\title{
Fano-Kondo Effect of Magnetic Impurity with Side-Coupled Graphene Flake
}

\author{
D. KRYCHOWSKI*, J. KOPIŃSKI, S. LIPIŃSKI \\ Institute of Molecular Physics, Polish Academy of Sciences M. Smoluchowskiego 17, 60-179 Poznań, Poland

\begin{abstract}
We examine transport through a spin-1/2 Kondo impurity, patterned between atomic scale electrodes in the presence of side-coupled nanographene flake. Impurity is attached to the edge of the flake, what allows to test the edge states. The Kotliar Ruckenstein slave boson approach is adopted to describe the strongly correlated impurity. We show that transport measurement of Fano-Kondo effect can serve as a spectroscopic probe of spin resolved graphene flake energy levels. The edge moments can be completely suppressed by gate voltage.
\end{abstract}

DOI: $10.12693 /$ APhysPolA.126.202

PACS: 73.22.Pr, 72.80.Vp, 73.23.-b, 72.15.Qm, 85.75.-d

\section{Introduction}

Among graphene materials, the nanostructures with zigzag edges has attracted considerable interest since these edges induce edge magnetism which originates from localized edge states [1]. For symmetric hexagonal nanoflakes discussed below with equal number of sites from both sublattices, a net magnetic moment vanishes [2]. The edge magnetism of graphene flakes has been recently discussed in [3] and has been also experimentally confirmed by scanning tunneling spectroscopy [4]. The aim of the present work is to investigate the impact of spin-polarized discrete level structure of graphene flake on the conductance of T-shape impurity-flake system in the Kondo range.

\section{Model}

The considered system is displayed in Fig. 1a. The magnetic impurity is attached to quantum wires and side coupled to hexagonal graphene flake with 864 carbon atoms. The flake states are described in unrestricted Hartree-Fock approximation by:

$$
\mathcal{H}_{\mathcal{G} \mathcal{F}}=-t_{0} \sum_{\langle i j\rangle \sigma} c_{i \sigma}^{\dagger} c_{j \sigma}+\sum_{i \sigma}\left[\mathcal{U}\left(\left\langle n_{i-\sigma}\right\rangle-\frac{1}{2}\right)+\mathcal{V}_{g}\right] n_{i \sigma},
$$

with nearest neighbor hopping integral $t_{0}=-2.5 \mathrm{eV}$ and graphene site Coulomb interaction $\mathcal{U}=1.2\left|t_{0}\right| . \mathcal{V}_{g}$ denotes the back gate voltage (in energy units) applied to graphene flake. Different nano-objects incorporating transition metal atoms can serve as spin $\frac{1}{2}$ impurity e.g. the divanadium molecule [5]. Impurity is modeled by Anderson Hamiltonian and we discuss the limit of infinite impurity Coulomb interaction, where double occupancy is forbidden. A very useful tool to describe strong correlations in this case is Kotliar-Ruckenstein slave boson representation [6] with $e$ and $p_{\sigma}$ slave boson operators representing empty or single occupied states of impurity

*corresponding author; e-mail: krychowski@ifmpan.poznan.pl
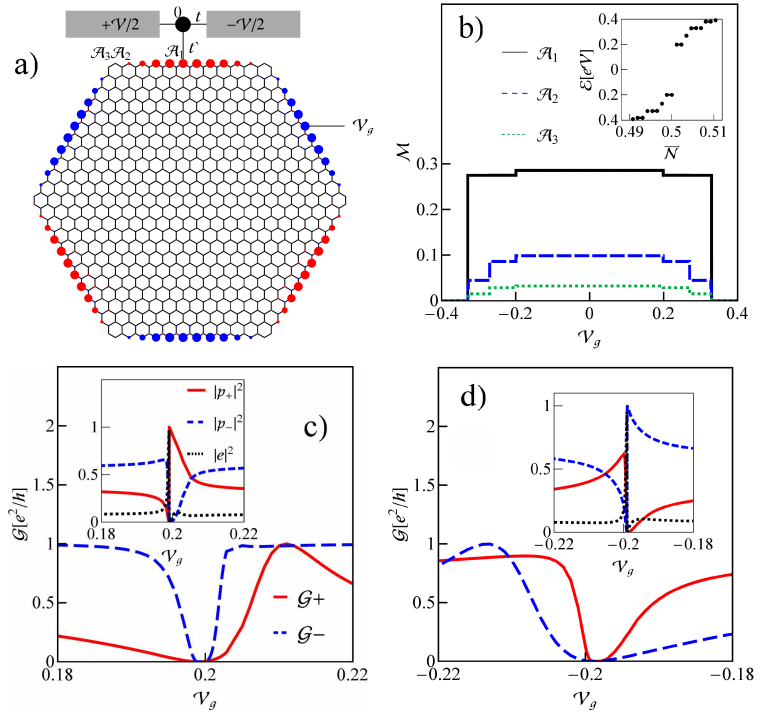

Fig. 1. Schematic view of the system (blue and red circles in graphene flake represent the negative and positive magnetic moment) b) back-gate voltage dependencies of local edge magnetic moments (inset shows energy spectrum as a function of average occupancy $\overline{\mathcal{N}}$ ) c, d) spin dependent linear conductancies plotted against $\mathcal{V}_{g}$ for impurity coupled with $\mathcal{A}_{1}$ atom (insets show corresponding slave bosons amplitudes).

respectively. In the mean field approximation (SB MFA) Hamiltonian of the discussed system reads:

$$
\begin{aligned}
\mathcal{H} & =\sum_{\sigma}\left(\mathcal{E}_{0}+\lambda_{\sigma}\right) n_{0 \sigma}+\lambda(\mathcal{I}-1)+\sum_{\sigma} \lambda_{\sigma}\left(\mathcal{Q}_{\sigma}-n_{0 \sigma}\right) \\
& +\sum_{l \alpha \sigma}\left[\left(t^{\prime} c_{l \sigma}^{\dagger}+t c_{k \alpha \sigma}^{\dagger}\right) z_{\sigma} f_{0 \sigma}+h . c .\right]+\mathcal{H}_{\mathcal{G} \mathcal{F}},
\end{aligned}
$$

where $l$ numbers the carbon atoms directly coupled to impurity, $\alpha=L, R$ labels the wires, $\mathcal{E}_{0}$ denotes impurity level and is choosen as $\mathcal{E}_{0}=-2 \mathrm{eV}, t, t^{\prime}$ represent hopping integrals to the wires and flake respectively $\left(t^{\prime}=0.5 \mathrm{eV}, t=2 t^{\prime}\right)$. The bandwidth of electrodes is assumed $\mathcal{D}=25 \mathrm{eV}$. The effective renormalized hopping in Eq. (2) is expressed by $z_{\sigma}^{\dagger} f_{0 \sigma}^{\dagger}\left(z_{\sigma} f_{0 \sigma}\right)$ with 
$z_{\sigma}=\left(e^{\dagger} p_{\sigma}\right) /\left(\sqrt{\mathcal{Q}_{\sigma}} \sqrt{1-\mathcal{Q}_{\sigma}}\right) . \quad \mathcal{Q}_{\sigma}$ denotes the charge (correspondence relation) and the completeness condition is included by the term with $\mathcal{I}$. The parameters $\lambda, \lambda_{\sigma}, e^{\dagger}(e)$ and $p_{\sigma}^{\dagger}\left(p_{\sigma}^{\dagger}\right)$ are determined by minimizing the MFA ground state of (2). The spin dependent linear conductances are defined as $\mathcal{G}_{\sigma}[\mathcal{V}=0]=\frac{e}{h^{2}} \mathcal{T}_{0 \sigma}(0)$, where $\mathcal{T}_{0 \sigma}(\omega)=-\left(\frac{\pi|t|^{2}}{\mathcal{D}}\left|z_{\sigma}\right|^{2}\right) \Im\left[G_{0 \sigma}^{R}(\omega)\right]$ represents the spin-dependent transmission and $G_{0 \sigma}^{R}$ is retarded Green's function of magnetic impurity. The polarization of conductance is given by $\mathcal{P}_{\mathcal{C}}=\left(\mathcal{G}_{+}-\mathcal{G}_{-}\right) /\left(\mathcal{G}_{+}+\mathcal{G}_{-}\right)$.

\section{Results and discussion}

Magnetic moments appear at the zigzag edges due to the localized character of the low energy states and the presence of the onsite Coulomb repulsion. The opposite sides are aligned antiparallel. The highest magnetic moment occurs in the centre of the sides ( $\mathcal{A}_{1}$ positions) and it results form dominant contribution of degenerate magnetic states with $\mathcal{E}= \pm 0.2 \mathrm{eV}$ energies. Figure $1 \mathrm{~b}$ presents gate voltage dependence of moments for the selected edge sites. Fermi level $\left(\mathcal{E}_{F}\right)$ shifts with the gate and each time the successive, discrete magnetic state crosses the Fermi level, the steps in local magnetization curves are observed. Increase of $\left|\mathcal{V}_{g}\right|$ reduces the edge contribution of the eigenstates of $\mathcal{E}_{F}$, above $\mathcal{V}_{g} \approx 0.33$ this share is negligible, the states do not exhibit spin polarization and nanoflake becomes nonmagnetic. For the chosen parameters, the impurity decoupled from the flake is almost in unitary Kondo regime. Attachment of the flake introduces interference effects with discrete energy channels and breaks the spin degeneracy at the impurity.
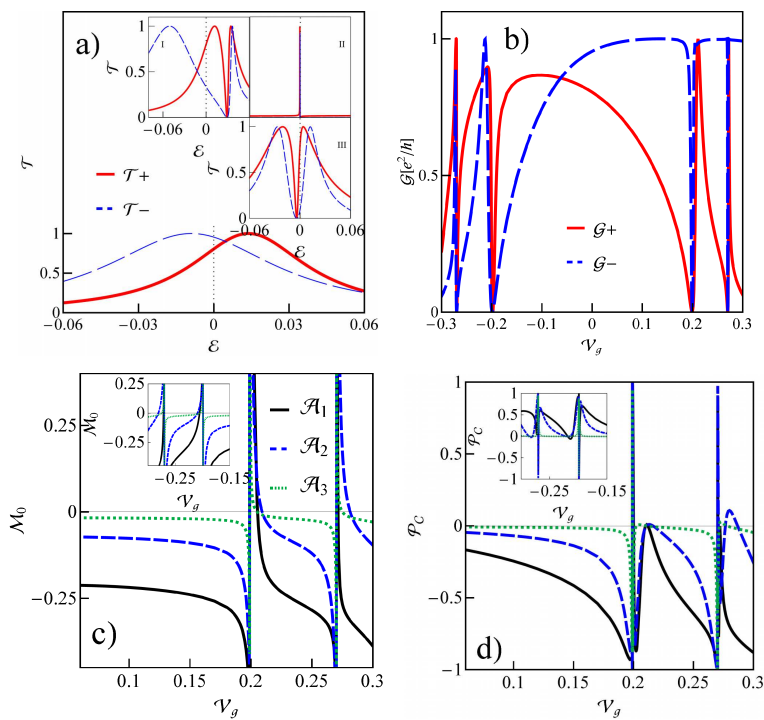

Fig. 2. Spin dependent transmissions for different back-gate voltages $\left(\mathcal{V}_{g}=\right.$ $0,-0.17(I),-0.1991(I I),-0.203(I I I)) \quad$ b) spin dependent conductance for impurity coupled with $\mathcal{A}_{1}$ atom c, d) back-gate voltage dependencies of impurity magnetic moments $\left(\mathcal{M}_{0}\right)$ and polarizations of conductance $\left(\mathcal{P}_{\mathcal{C}}\right)$ for three different attachments of the flake.
Figures 1c, 1d and 2a, 2b illustrate the gate dependencies of conductances, expectation values of slave boson operators and representative transmissions for impurity coupled with $\mathcal{A}_{1}$ atom. For chemical potential located not to close to any discrete level of the flake, the shape of Kondo resonance is only weakly perturbed, but it is spin splitted by the presence of magnetic atoms directly coupled to impurity, what reflects in polarization of conductance. Moving with $\mathcal{E}_{\mathcal{F}}$ closer to the position of a discrete level (e.g. $\mathcal{V}_{g}=-0.17$ ), a pronounced dip in transmission emerges as a consequence of destructive interference, for energy corresponding to flake energy level. Moving even closer, interference effects destroy the Kondo resonance $\left(\mathcal{V}_{g}=-0.1991,\left|p_{+}\right|^{2}=0.041,\left|p_{-}\right|^{2}=0.004\right.$, $\left.|e|^{2}=0.954\right)$, and for Fermi level slightly above the singularity, the Fano-Kondo dip centered almost at the chemical potential is visible.

The presented changes of magnetizations and polarizations for $\mathcal{A}_{1}, \mathcal{A}_{2}$, and $\mathcal{A}_{3}$ positions are remarkable (Fig. 2c, 2d). Outside the positions of discrete levels, impurity moment is larger, the higher is moment of the probed edge carbon atom. When discrete level crosses the chemical potential, magnetic moments and polarizations dramatically increase and flip. This phenomena might be of interest for spintronic applications, especially due to the fact that they occur within extremely narrow gate voltage ranges, dictated by the weak coupling strength of impurity to the flake. The presented results highlight the way of probing the energy structure of nanoflakes by measuring conductance. The conductance dips occur in the position of discrete energy levels of the examined flake and polarization of conductance of impurity contacted with $\mathcal{A}_{1}$ atom is roughly proportional, but of opposite sign, to the polarization of the probed flake atom.

\section{Acknowledgments}

This work was supported by the Polish Ministry of Science and Higher Education as a research Project No. N N202 199239 for years 2010-2013.

\section{References}

[1] Y.-W. Son, M. L. Cohen, S.G. Louie, Nature 444, 347 (2006).

[2] E. H. Lieb, Phys. Rev. Lett. 62, 1201 (1989).

[3] I. Weymann, J. Barnas, S. Krompiewski, Phys. Rev. B 85, 205306 (2012).

[4] Ch. Tao, L. Jiao, O.V. Yazyev, Y.-C. Chen, J. Feng, X. Zhang, R.B. Capaz, J.M. Tour, A. Zettl, S.G. Louie, H. Dai, M.F. Crommie, Nature Phys. 7, 616 (2011).

[5] W. Liang, M.P. Shores, M. Bockrath, J.R. Long, H. Park, Nature 417, 725 (2002).

[6] G. Kotliar, A.E. Ruckenstein, Phys. Rev. Lett. 57, 1362 (1986). 